\title{
Uncertainty analysis of the coefficients of friction during the tightening process of bolted joints
}

\author{
Arthur Seibel ${ }^{*}$, Andreas Japing and Josef Schlattmann
}

*Correspondence:

arthur.seibel@tuhh.de

Workgroup on System

Technologies and Engineering

Design Methodology, Hamburg

University of Technology, 21073

Hamburg, Germany

\begin{abstract}
In this paper, we introduce closed-form symbolic expressions for the possibility distributions of the coefficients of friction during the tightening process of bolted joints. The parameters in the distribution functions are then identified by a standardized fastener testing system according to ISO 16047. An uncertainty analysis finally shows that the total amount of uncertainty in the coefficient of bearing friction is almost $40 \%$ larger than in the coefficient of thread friction. Furthermore, the real value of the coefficent of bearing friction is likely to be about $5 \%$ higher than the expected value.
\end{abstract}

Keywords: Bolted joints tribology; Coefficient of bearing friction; Coefficient of thread friction; Uncertainty analysis; Possibility theory

\section{Introduction}

Bolted joints are among the most used joints in mechanical engineering. But failures still occur during the tightening process and in operation. In order to improve the quality and reliability of bolted joints, a main goal is to achieve a proper clamping force. With a certain effort [1], it is possible to measure the clamping force directly. But most of the bolts and screws still are assembled by an indirect measurement of the clamping force [2].

The required torque $T$ for tightening a bolted joint is determined by [3]

$$
T=T_{\mathrm{b}}+T_{\mathrm{t}},
$$

where $T_{\mathrm{b}}$ denotes the bearing friction torque component and $T_{\mathrm{t}}$ the thread friction torque component. These components are in a direct relationship with the clamping force and the coefficients of friction of the particular material pairing. Hence, the information about the coefficients of friction has a great influence on the quality and reliability of bolted joint connections.

Suppliers of bolts and screws often give only parameter windows for the desired coefficients of friction. These intervals are rather general and may not be suitable for individual applications. Furthermore, no information about the distribution functions of the coefficients of friction is provided.

Basically, two main approaches for modeling distribution functions are available: probability [4] and possibility theory [5]. In probability theory, the integral over the distribution function $p(x)$ is equal to one:

(c) 2014 Seibel et al.; licensee Springer. This is an Open Access article distributed under the terms of the Creative Commons Attribution License (http://creativecommons.org/licenses/by/4.0), which permits unrestricted use, distribution, and reproduction in any medium, provided the original work is properly credited. 


$$
\int_{-\infty}^{+\infty} p(x) \mathrm{d} x=1,
$$

whereas in possibility theory, the distribution functions $\pi(x)$ are always normalized:

$$
\max \{\pi(x)\}=1 .
$$

For example, the Gaussian distribution function can be generally expressed by

$$
f(x)=C \exp \left[-\frac{1}{2}\left(\frac{x-\bar{x}}{\sigma}\right)^{2}\right],
$$

where $\bar{x}$ denotes the mean value and $\sigma$ the standard deviation of $f(x)$. In probability theory, the constant scaling factor is set to

$$
C=\frac{1}{\sigma \sqrt{2 \pi}}
$$

to satisfy the property (2). However, in possibility theory, we set

$$
C=1
$$

to meet the requirement (3).

The general disadvantage in working with probability distribution functions is that in order to construct a probabilistic model, a large amount of statistical data is needed, which, in reality, is not always the case. In contrast to that, when only little information about the uncertain parameters is available, possibility theory is most appropriate. For this reason, we use possibility theory in this paper to model parametric uncertainty.

The main contributions of this paper are the following. First, closed-form symbolic expressions for the possibility distributions of the coefficients of friction during the tightening process of bolted joints are introduced for the first time. The parameters in the distribution functions are then identified by a standardized fastener testing system according to ISO 16047. An uncertainty analysis finally compares the total amount of uncertainty in the coefficient of bearing friction with that in the coefficient of thread friction. Furthermore, the deviations of the real values of the coefficients of friction from the expected values are given.

\section{Possibility theory}

Historically, possibility theory emerged from the theory of fuzzy sets [6] because of 'the need for a systematic way of dealing with [...] forms of uncertainty which are not probabilistic in nature' [5]. In fact, the membership function $\mu_{\tilde{x}}(x)$ of a fuzzy number $\tilde{x}$ can be viewed as a possibility distribution $\pi(x)$ of the parameter $x$ [7]. More specifically, the possibility distribution $\pi(x)$ is a function that maps a value $x$ to the possibility of the singleton event $\{x\}[8]$ :

$$
\pi(x)=\Pi(\{x\}) .
$$

However, a high possibility of some event does not automatically mean that this event has also a high probability. In fact, the probability $P(A)$ of an event $A$ can be viewed as a lower bound for the corresponding possibility $\Pi(A)[9]$ :

$$
\Pi(A) \geq P(A) .
$$

This is in accordance with intuition since 'before an event becomes probable, it must be possible' [8]. For a detailed overview of possibility theory, the reader is referred to [5]. 


\section{Sensitivity analysis}

For the analysis of models with uncertain parameters, it is important to know to which extent the uncertainties of the individual model parameters contribute to the overall uncertainty of the model answer. Such a sensitivity analysis can be defined by the total differential [10]

$$
\mathrm{d} f=\sum_{i=1}^{n}(\mathrm{~d} f)_{i}=\sum_{i=1}^{n} \frac{\partial f(\overline{\boldsymbol{x}})}{\partial x_{i}} \mathrm{~d} x_{i}
$$

of the model function $f$ at the point $\bar{x}=\left(\bar{x}_{1}, \ldots, \bar{x}_{n}\right)$, where $\mathrm{d} f$ represents the total change of $f$ in a neighborhood of $\bar{x}$ if all parameters are changed simultaneously. The change $\mathrm{d} x_{i}$ of a single parameter $x_{i}$ contributes to the amount $(\mathrm{d} f)_{i}$ to the total change $\mathrm{d} f$.

If the change rates $\mathrm{d} x_{i}$ of the parameters $x_{i}$ are assumed to be a constant fraction $c$ of the corresponding modal values $\bar{x}_{i}$, the total differential from Equation (4) can be written as [10]

$$
\mathrm{d} f=c \sum_{i=1}^{n} \frac{\partial f(\overline{\boldsymbol{x}})}{\partial x_{i}} \bar{x}_{i} .
$$

Using Equation (5), we can define the following relative sensitivity measures [10]:

$$
\rho_{i}=\frac{\left|\frac{\partial f(\bar{x})}{\partial x_{i}}\right| \bar{x}_{i}}{\sum_{j=1}^{n}\left|\frac{\partial f(\bar{x})}{\partial x_{j}}\right| \bar{x}_{j}}
$$

satisfying the consistency condition

$$
\sum_{i=1}^{n} \rho_{i}=1 .
$$

\section{Uncertainty propagation}

In order to propagate the uncertainties through the computations, we introduce the following transformation:

Let

$$
\pi(x)= \begin{cases}\pi^{\mathrm{L}}(x), & x \leq \bar{x}, \\ \pi^{\mathrm{R}}(x), & x>\bar{x},\end{cases}
$$

be the possibility distribution of the parameter $x$ in the $x$ domain, where $\pi^{\mathrm{L}}(x)$ denotes the left branch, $\pi^{\mathrm{R}}(x)$ the right branch, and $\bar{x}$ the modal value of $\pi(x)$. The transformation of $\pi(x)$ into the $\pi$ domain leads to the (generalized) interval $x(\pi)=\left[x^{\mathrm{L}}(\pi), x^{\mathrm{R}}(\pi)\right]$ with

$$
\begin{array}{ll}
x^{\mathrm{L}}(\pi)=\pi^{\mathrm{L}}(x)^{-1}, & 0<\pi \leq 1, \\
x^{\mathrm{R}}(\pi)=\pi^{\mathrm{R}}(x)^{-1}, & 0<\pi \leq 1 .
\end{array}
$$

For example, the transformation of the Gaussian possibility distribution leads to

$$
\begin{array}{ll}
x^{\mathrm{L}}(\pi)=\bar{x}-\sigma \sqrt{-2 \ln (\pi)}, & 0<\pi \leq 1, \\
x^{\mathrm{R}}(\pi)=\bar{x}+\sigma \sqrt{-2 \ln (\pi)}, & 0<\pi \leq 1 .
\end{array}
$$

The Gaussian possibility distribution in the $x$ and the $\pi$ domain is illustrated in Figure 1 . 

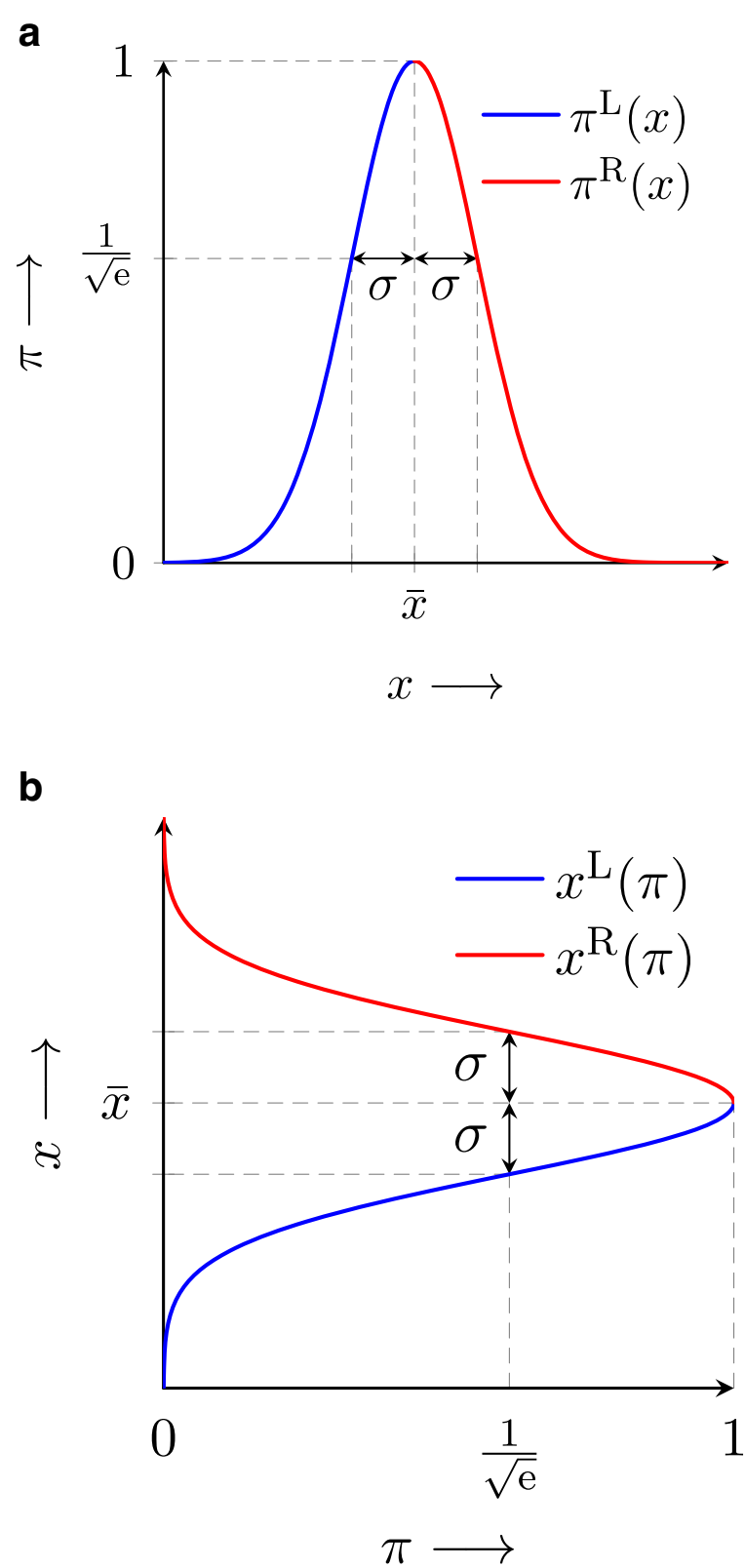

Figure 1 Gaussian possibility distribution. (a) $x$ domain. (b) $\pi$ domain.

Using the above transformation, we can formulate the following analytical approach [11]: Let $\pi\left(x_{1}\right), \ldots, \pi\left(x_{n}\right)$ be the possibility distributions of the $n$ independent parameters $x_{1}, \ldots, x_{n}$, and let $f: \mathbb{R}^{n} \rightarrow \mathbb{R}$ be a continuous function with $y=f\left(x_{1}, \ldots, x_{n}\right)$. Furthermore, let $f$ be (strictly) monotonic increasing in $x_{i}, i=1, \ldots, k$, and (strictly) monotonic decreasing in $x_{j}, j=k+1, \ldots, n$, in the domain of interest. Then, the possibility distribution $y(\pi)=\left[y^{\mathrm{L}}(\pi), y^{\mathrm{R}}(\pi)\right]$ of $y$ in the $\pi$ domain is determined by

$$
\begin{array}{ll}
y^{\mathrm{L}}(\pi)=f\left(x_{1}^{\mathrm{L}}(\pi), \ldots, x_{k}^{\mathrm{L}}(\pi), x_{k+1}^{\mathrm{R}}(\pi), \ldots, x_{n}^{\mathrm{R}}(\pi)\right), & 0<\pi \leq 1, \\
y^{\mathrm{R}}(\pi)=f\left(x_{1}^{\mathrm{R}}(\pi), \ldots, x_{k}^{\mathrm{R}}(\pi), x_{k+1}^{\mathrm{L}}(\pi), \ldots, x_{n}^{\mathrm{L}}(\pi)\right), & 0<\pi \leq 1 .
\end{array}
$$


If Equations (6) are invertible with respect to $\pi$, then the possibility distribution of $y$ in the $y$ domain yields

$$
\pi(y)= \begin{cases}y^{\mathrm{L}}(\pi)^{-1}, & y^{\mathrm{L}}(0)<y \leq y^{\mathrm{L}}(1), \\ y^{\mathrm{R}}(\pi)^{-1}, & y^{\mathrm{R}}(1)<y<y^{\mathrm{R}}(0) .\end{cases}
$$

\section{Distribution functions}

In this section, we use the above analytical approach to derive closed-form symbolic expressions for the distribution functions of the coefficients of friction during the tightening process of bolted joints.

\section{Coefficient of bearing friction}

The coefficient of friction between the turning head or nut and the bearing surface $\mu_{\mathrm{b}}$ can be computed from [3]

$$
\mu_{\mathrm{b}}=\frac{T_{\mathrm{b}}}{F r_{\mathrm{b}}}
$$

where $T_{\mathrm{b}}$ denotes the bearing friction torque component, $F$ the clamping force, and $r_{\mathrm{b}}$ the effective bearing radius.

During the tightening process, the clamping force $F$ should be achieved exactly. Hence, it exhibits no uncertainty. The parameters $T_{\mathrm{b}}$ and $r_{\mathrm{b}}$, on the other hand, are assumed to be normally distributed.

With the partial derivatives

$$
\begin{aligned}
& \frac{\partial \mu_{\mathrm{b}}}{\partial T_{\mathrm{b}}}=+\frac{1}{F r_{\mathrm{b}}}, \\
& \frac{\partial \mu_{\mathrm{b}}}{\partial r_{\mathrm{b}}}=-\frac{T_{\mathrm{b}}}{F r_{\mathrm{b}}^{2}},
\end{aligned}
$$

the sensitivity measures for $\mu_{\mathrm{b}}$ are

$$
\rho_{T_{\mathrm{b}}}=\rho_{r_{\mathrm{b}}}=0.5 \text {. }
$$

Hence, the uncertainties of $T_{\mathrm{b}}$ and $r_{\mathrm{b}}$ contribute to the same amount to the overall uncertainty of $\mu_{\mathrm{b}}$.

We can see from the signs of the partial derivatives that $\mu_{\mathrm{b}}$ is (strictly) monotonic increasing in $T_{\mathrm{b}}$ and (strictly) monotonic decreasing in $r_{\mathrm{b}}$ for positive values. Hence, according to Equations (6), the possibility distribution of $\mu_{\mathrm{b}}$ in the $\pi$ domain is $\mu_{\mathrm{b}}(\pi)=$ $\left[\mu_{\mathrm{b}}^{\mathrm{L}}(\pi), \mu_{\mathrm{b}}^{\mathrm{R}}(\pi)\right]$ with

$$
\begin{array}{ll}
\mu_{\mathrm{b}}^{\mathrm{L}}(\pi)=\frac{T_{\mathrm{b}}^{\mathrm{L}}(\pi)}{F r_{\mathrm{b}}^{\mathrm{R}}(\pi)}=\frac{\bar{T}_{\mathrm{b}}-\sigma_{T_{\mathrm{b}}} \sqrt{-2 \ln (\pi)}}{F\left(\bar{r}_{\mathrm{b}}+\sigma_{r_{\mathrm{b}}} \sqrt{-2 \ln (\pi)}\right)}, & 0<\pi \leq 1, \\
\mu_{\mathrm{b}}^{\mathrm{R}}(\pi)=\frac{T_{\mathrm{b}}^{\mathrm{R}}(\pi)}{F r_{\mathrm{b}}^{\mathrm{L}}(\pi)}=\frac{\bar{T}_{\mathrm{b}}+\sigma_{T_{\mathrm{b}}} \sqrt{-2 \ln (\pi)}}{F\left(\bar{r}_{\mathrm{b}}-\sigma_{r_{\mathrm{b}}} \sqrt{-2 \ln (\pi)}\right)}, & 0<\pi \leq 1,
\end{array}
$$

and in the $\mu_{\mathrm{b}}$ domain,

$$
\pi\left(\mu_{\mathrm{b}}\right)=\exp \left[-\frac{1}{2}\left(\frac{F \bar{r}_{\mathrm{b}} \mu_{\mathrm{b}}-\bar{T}_{\mathrm{b}}}{F \sigma_{r_{\mathrm{b}}} \mu_{\mathrm{b}}+\sigma_{T_{\mathrm{b}}}}\right)^{2}\right] .
$$


Introducing the abbreviations

$$
\begin{aligned}
\bar{\mu}_{\mathrm{b}} & =\frac{\bar{T}_{\mathrm{b}}}{F \bar{r}_{\mathrm{b}}}, \\
\sigma_{\mu_{\mathrm{b}}} & =\frac{\sigma_{T_{\mathrm{b}}}}{F \sigma_{r_{\mathrm{b}}}},
\end{aligned}
$$

Equation (7) can be simplified to

$$
\pi\left(\mu_{\mathrm{b}}\right)=\exp \left[-\frac{1}{2} \frac{\bar{r}_{\mathrm{b}}^{2}}{\sigma_{r_{\mathrm{b}}}^{2}}\left(\frac{\mu_{\mathrm{b}}-\bar{\mu}_{\mathrm{b}}}{\mu_{\mathrm{b}}+\sigma_{\mu_{\mathrm{b}}}}\right)^{2}\right] .
$$

\section{Coefficient of thread friction}

For metric ISO threads with a thread profile angle of $60^{\circ}$, the coefficient of friction between male and female threads $\mu_{\mathrm{t}}$ can be approximated by [3]

$$
\mu_{\mathrm{t}}=\frac{\sqrt{3}}{d_{\mathrm{t}}}\left(\frac{T_{\mathrm{t}}}{F}-\frac{P}{2 \pi}\right)
$$

where $d_{\mathrm{t}}$ denotes the effective thread diameter, $T_{\mathrm{t}}$ the thread friction torque component, $F$ the clamping force, and $P$ the thread pitch. (Note that the possibility degree $\pi$, which is used throughout the paper, should not be confused with the mathematical constant $\pi=3.14 \ldots)$.

Again, the clamping force $F$ is assumed to exhibit no uncertainty, and the other parameters are assumed to be normally distributed.

With the partial derivatives

$$
\begin{aligned}
& \frac{\partial \mu_{\mathrm{t}}}{\partial d_{\mathrm{t}}}=-\frac{\sqrt{3}}{d_{\mathrm{t}}^{2}}\left(\frac{T_{\mathrm{t}}}{F}-\frac{P}{2 \pi}\right), \\
& \frac{\partial \mu_{\mathrm{t}}}{\partial T_{\mathrm{t}}}=+\frac{\sqrt{3}}{d_{\mathrm{t}}} \frac{1}{F}, \\
& \frac{\partial \mu_{\mathrm{t}}}{\partial P}=-\frac{\sqrt{3}}{d_{\mathrm{t}}} \frac{1}{2 \pi},
\end{aligned}
$$

the sensitivity measures for $\mu_{\mathrm{t}}$ are

$$
\begin{aligned}
\rho_{d_{\mathrm{t}}} & =\frac{2 \pi \bar{T}_{\mathrm{t}}-F \bar{P}}{4 \pi \bar{T}_{\mathrm{t}}}, \\
\rho_{T_{\mathrm{t}}} & =0.5, \\
\rho_{P} & =\frac{F \bar{P}}{4 \pi \bar{T}_{\mathrm{t}}},
\end{aligned}
$$

with

$$
\rho_{d_{\mathrm{t}}}+\rho_{P}=0.5
$$

However, since $2 \pi \bar{T}_{\mathrm{t}} \gg F \bar{P}$, we can deduce:

$$
\begin{aligned}
\rho_{d_{\mathrm{t}}} & \approx 0.5, \\
\rho_{T_{\mathrm{t}}} & =0.5, \\
\rho_{P} & \approx 0 .
\end{aligned}
$$

Hence, the uncertainties of $d_{\mathrm{t}}$ and $T_{\mathrm{t}}$ contribute to the nearly same amount to the overall uncertainty of $\mu_{\mathrm{t}}$, whereas the uncertainty of $P$ has a negligible influence.

We can see from the signs of the partial derivatives that $\mu_{\mathrm{t}}$ is (strictly) monotonic increasing in $T_{\mathrm{t}}$ and (strictly) monotonic decreasing in $d_{\mathrm{t}}$ for positive values. Hence, 
according to Equations (6), the possibility distribution of $\mu_{\mathrm{t}}$ in the $\pi$ domain is $\mu_{\mathrm{t}}(\pi)=$ $\left[\mu_{\mathrm{t}}^{\mathrm{L}}(\pi), \mu_{\mathrm{t}}^{\mathrm{R}}(\pi)\right]$ with

$$
\begin{array}{rlrl}
\mu_{\mathrm{t}}^{\mathrm{L}}(\pi) & =\frac{\sqrt{3}}{d_{\mathrm{t}}^{\mathrm{R}}(\pi)}\left(\frac{T_{\mathrm{t}}^{\mathrm{L}}(\pi)}{F}-\frac{P}{2 \pi}\right) & \\
& =\frac{\sqrt{3}}{2 \pi F} \frac{\left(2 \pi \bar{T}_{\mathrm{t}}-F P\right)-2 \pi \sigma_{T_{\mathrm{t}}} \sqrt{-2 \ln (\pi)}}{\bar{d}_{\mathrm{t}}+\sigma_{d_{\mathrm{t}} \sqrt{-2 \ln (\pi)}}}, & & 0<\pi \leq 1, \\
\mu_{\mathrm{t}}^{\mathrm{R}}(\pi) & =\frac{\sqrt{3}}{d_{\mathrm{t}}^{\mathrm{L}}(\pi)}\left(\frac{T_{\mathrm{t}}^{\mathrm{R}}(\pi)}{F}-\frac{P}{2 \pi}\right) & & \\
& =\frac{\sqrt{3}}{2 \pi F} \frac{\left(2 \pi \bar{T}_{\mathrm{t}}-F P\right)+2 \pi \sigma_{T_{\mathrm{t}}} \sqrt{-2 \ln (\pi)}}{\bar{d}_{\mathrm{t}}-\sigma_{d_{\mathrm{t}}} \sqrt{-2 \ln (\pi)}}, & & 0<\pi \leq 1,
\end{array}
$$

and in the $\mu_{\mathrm{t}}$ domain,

$$
\pi\left(\mu_{\mathrm{t}}\right)=\exp \left[-\frac{1}{2}\left(\frac{a \mu_{\mathrm{t}}^{2}+b \mu_{\mathrm{t}}+c}{d \mu_{\mathrm{t}}^{2}+e \mu_{\mathrm{t}}+f}\right)\right]
$$

with

$$
\begin{aligned}
& a=4 \pi^{2} \bar{d}_{\mathrm{t}}^{2} F^{2}, \\
& b=-4 \sqrt{3} \pi \bar{d}_{\mathrm{t}} F\left(2 \pi \bar{T}_{\mathrm{t}}-F P\right), \\
& c=12 \pi^{2} \bar{T}_{\mathrm{t}}^{2}-12 \pi \bar{T}_{\mathrm{t}} F P+3 F^{2} P^{2}, \\
& d=4 \pi^{2} \sigma_{\mathrm{d}_{\mathrm{t}}}^{2} F^{2}, \\
& e=8 \sqrt{3} \pi^{2} \sigma_{d_{\mathrm{t}}} \sigma_{T_{\mathrm{t}}} F, \\
& f=12 \pi^{2} \sigma_{T_{\mathrm{t}}}^{2} .
\end{aligned}
$$

\section{Parameter identification}

In order to identify the parameters in the distribution functions of $\mu_{\mathrm{b}}$ and $\mu_{\mathrm{t}}$, practical experiments had been carried out on a fastener testing system according to ISO 16047 [12] as illustrated in Figure 2. This system contains a multi-parameter sensor that enables a measurement of the fastener preload, the tightening torque, and one of the friction torque components at the same time.

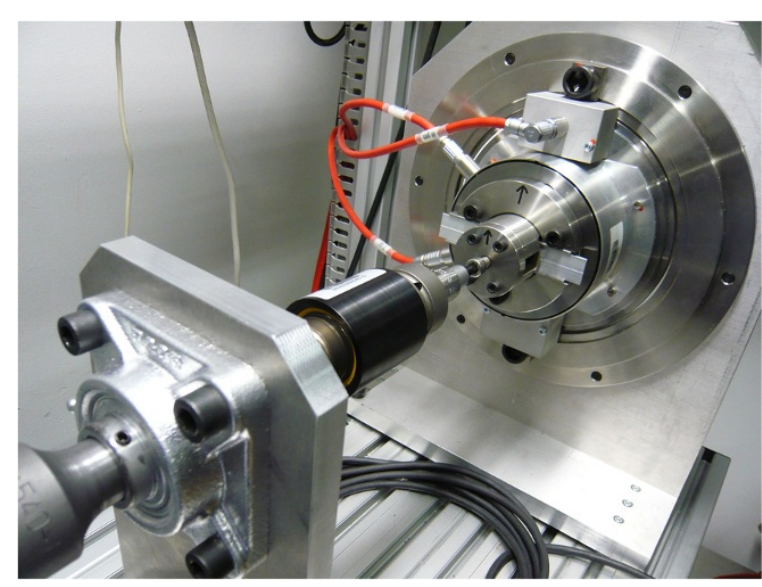

Figure 2 Fastener testing system used in our experiments. 


\section{Materials and methods}

The descriptions and materials of the test parts are summarized in Table 1. The dimensions of the flat steel bar are illustrated in Figure 3.

For carrying out the experiments, we followed the instructions from ISO 16047 [12]. A tightening torque was steadily applied until a clamping force of $16 \mathrm{kN}$ was reached. Here, $F, T$, and $T_{\mathrm{b}}$ were measured, and $T_{\mathrm{t}}$ was computed according to Equation (1). The sample size was 32 bolts.

\section{Experimental results}

Since the effective bearing radius is usually hard to determine in practice [13], $r_{\mathrm{b}}$ is assumed to be normally distributed with the mean value

$$
\bar{r}_{\mathrm{b}}=\frac{d_{\mathrm{o}}+d_{\mathrm{i}}}{4}=6.625 \mathrm{~mm}
$$

and the standard deviation

$$
\sigma_{r_{\mathrm{b}}}=\frac{d_{\mathrm{o}}-d_{\mathrm{i}}}{12}=0.625 \mathrm{~mm},
$$

where $d_{\mathrm{o}}$ denotes the outer diameter and $d_{\mathrm{i}}$ the inner diameter of the bearing surface.

Furthermore, the effective thread diameter is also usually hard to determine in practice [13]. For this reason, $d_{\mathrm{t}}$ is also assumed to be normally distributed with the mean value

$$
\bar{d}_{\mathrm{t}}=d_{2}=7.188 \mathrm{~mm}
$$

and the standard deviation

$$
\sigma_{d_{\mathrm{t}}}=\frac{d-D_{1}}{6}=0.226 \mathrm{~mm},
$$

where $d_{2}$ denotes the thread pitch diameter, $d$ the nominal thread diameter, and $D_{1}$ the minor nut thread diameter. The standard deviations are chosen such that the intervals $\left[d_{\mathrm{i}}, d_{\mathrm{o}}\right]$ and $\left[D_{1}, d\right]$ correspond to $6 \cdot 2 \sigma_{r_{\mathrm{b}}}$ and $6 \sigma_{d_{\mathrm{t}}}$, respectively. (The factor two at $\sigma_{r_{\mathrm{b}}}$ results from the fact that the standard deviation of a diameter is twice as large as the standard deviation of the corresponding radius). Beyond the interval boundaries, the possibility values are smaller than $1 \%$ [14] and can be thus neglected. In fact, they are physically impossible.

Using the above assumptions, the distribution functions of $\mu_{\mathrm{b}}$ and $\mu_{\mathrm{t}}$ for $F \in[2,16]$ $\mathrm{kN}$ are illustrated in Figure 4. We can see that after an initial shakedown, a steady-state distribution is always reached.

According to ISO 16047 [12], the determination of the coefficients of friction shall be carried out at $75 \%$ of the maximum clamping force. In our experiments, this is

Table 1 Descriptions and materials of the test parts

\begin{tabular}{lcr}
\hline Test part & Description/material & Remark \\
\hline Bolt & DIN 6921 M8 $\times 5010.9$ & Black chromated \\
Nut & DIN 934 M8 8.8 & Black chromated \\
Flat bar & S235JRC+C (EN 1.0122) & Surface polished \\
\hline
\end{tabular}




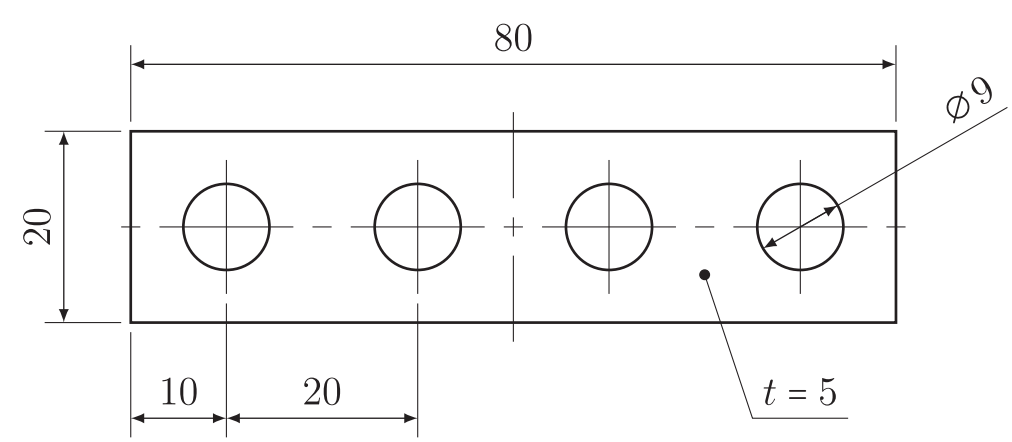

Figure 3 Dimensions of the flat steel bar. Here, $t$ denotes the thickness.

the case at $F=12 \mathrm{kN}$. The distribution functions of $\mu_{\mathrm{b}}$ and $\mu_{\mathrm{t}}$ at this point are

$$
\begin{aligned}
& \pi\left(\mu_{\mathrm{b}}\right)=\exp \left[-56\left(\frac{\mu_{\mathrm{b}}-0.114}{\mu_{\mathrm{b}}+0.091}\right)^{2}\right], \\
& \pi\left(\mu_{\mathrm{t}}\right)=\exp \left[-\frac{1}{2}\left(\frac{2.94 \cdot 10^{5} \mu_{\mathrm{t}}^{2}-1.13 \cdot 10^{5} \mu_{\mathrm{t}}+0.109 \cdot 10^{5}}{7.19 \cdot 10^{-3} \mu_{\mathrm{t}}^{2}+152 \mu_{\mathrm{t}}+19.9}\right)\right] .
\end{aligned}
$$

The plots of Equations (8) are illustrated in Figure 5.
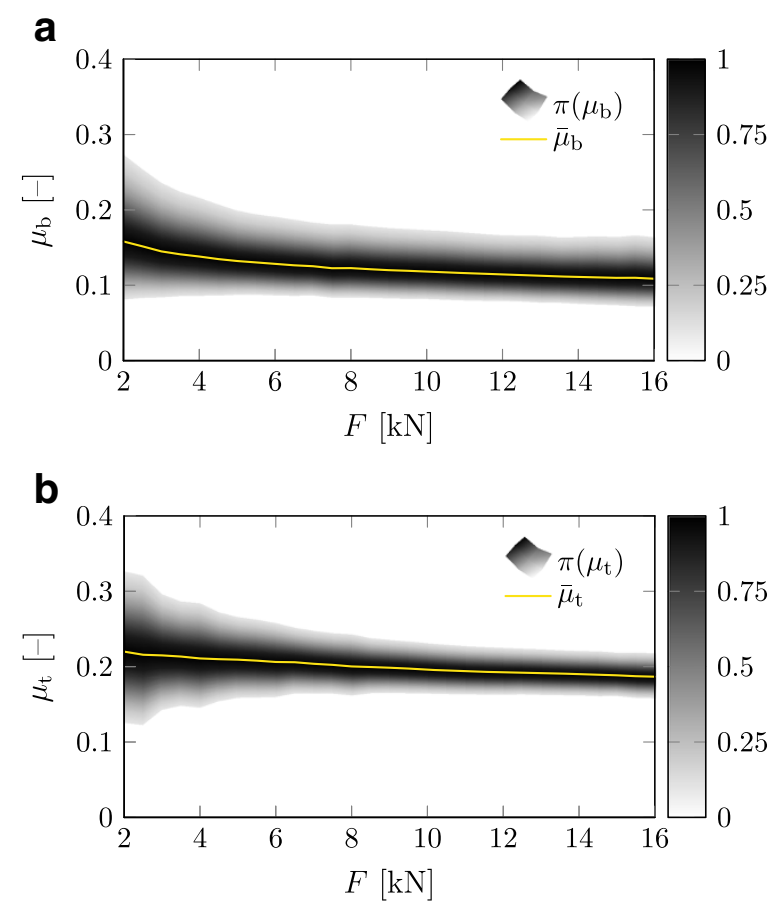

Figure 4 Distribution functions of $\mu_{\mathrm{b}}$ and $\mu_{\mathrm{t}}$ for $F \in[2,16] \mathrm{kN}$. (a) Distribution function of $\mu_{\mathrm{b}}$. (b) Distribution function of $\mu_{\mathrm{t}}$. 


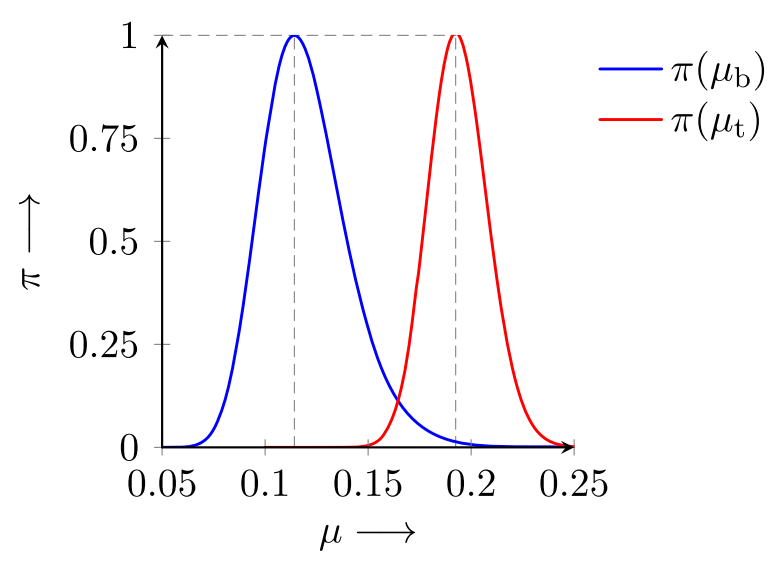

Figure 5 Distribution functions of $\mu_{\mathrm{b}}$ and $\mu_{\mathrm{t}}$ at $F=12 \mathrm{kN}$.

\section{Uncertainty analysis}

In order to quantify the total amount of uncertainty of a possibility distribution $\pi(x)$, we use the (absolute) cardinality [14]:

$$
\operatorname{card}(\pi(x))=\int_{-\infty}^{+\infty} \pi(x) \mathrm{d} x
$$

Since the coefficients of friction are all elements of $[0,1]$, the total amount of uncertainty can be defined as

$$
\operatorname{uncer}(\pi(x))=\int_{0}^{1} \pi(x) \mathrm{d} x .
$$

Numerical integration of Equations (8) yields

$$
\begin{aligned}
& \operatorname{uncer}\left(\pi\left(\mu_{\mathrm{b}}\right)\right)=0.050, \\
& \operatorname{uncer}\left(\pi\left(\mu_{\mathrm{t}}\right)\right)=0.036,
\end{aligned}
$$

meaning that in our application, the total amount of uncertainty in $\mu_{\mathrm{b}}$ is almost $40 \%$ larger than in $\mu_{\mathrm{t}}$.

In the next step, the (relative) eccentricities of the possibility distributions of the coefficients of friction are analyzed [14]:

$$
\operatorname{ecc}(\pi(x))=\frac{\operatorname{defuzz}(\pi(x))-\bar{x}}{\bar{x}} .
$$

Here,

$$
\operatorname{defuzz}(\pi(x))=\frac{\int_{0}^{1} x \pi(x) \mathrm{d} x}{\int_{0}^{1} \pi(x) \mathrm{d} x}
$$

denotes the defuzzified value of $\pi(x)$.

Further numerical integration yields

$$
\begin{aligned}
& \operatorname{defuzz}\left(\pi\left(\mu_{\mathrm{b}}\right)\right)=0.120, \\
& \operatorname{defuzz}\left(\pi\left(\mu_{\mathrm{t}}\right)\right)=0.194 .
\end{aligned}
$$


Hence, the desired eccentricities are

$$
\begin{aligned}
& \operatorname{ecc}\left(\pi\left(\mu_{\mathrm{b}}\right)\right)=\frac{0.120-0.114}{0.114}=0.05, \\
& \operatorname{ecc}\left(\pi\left(\mu_{\mathrm{t}}\right)\right)=\frac{0.194-0.193}{0.193}=0.01 .
\end{aligned}
$$

Both eccentricities are positive, that is, the real values of the coefficients of friction will be potentially higher than the expected values. More specifically, the real value of $\mu_{\mathrm{b}}$ is likely to be about $5 \%$ and the real value of $\mu_{\mathrm{t}}$ to be about $1 \%$ higher than the corresponding expected value.

\section{Conclusions}

We introduced closed-form symbolic expressions for the possibility distributions of the coefficients of friction during the tightening process of bolted joints. This relieves the engineer from the burden of propagating the uncertainties through the computations to obtain the uncertain output. The parameters in the distribution functions can be identified by a standardized fastener testing system according to ISO 16047 as has been demonstrated in this paper. An uncertainty analysis also revealed that the total amount of uncertainty in the coefficient of bearing friction is almost $40 \%$ larger than in the coefficient of thread friction. This finding suggests that in practice, it is more important to control the coefficient of bearing friction than the coefficient of thread friction. The uncertainty analysis also showed that the real value of the coefficient of bearing friction is likely to be about $5 \%$ and the real value of the coefficient of thread friction to be about $1 \%$ higher than the corresponding expected value.

\section{Competing interests}

The authors declare that they have no competing interests.

Received: 18 February 2014 Accepted: 16 September 2014

Published online: 03 October 2014

\section{References}

1. Baker, R: Ultrasonic tightening. Sealing Technol. 2011(8), 10-12 (2011)

2. Kloos, K-H, Thomala, W: Schraubenverbindungen: Grundlagen, Berechnung, Eigenschaften, Handhabung. Springer, Berlin (2007)

3. Kellermann, R, Klein, H-C: Untersuchungen über den Einfluß der Reibung auf Vorspannung und Anzugsmoment von Schraubenverbindungen. Konstruktion. 7(2), 54-68 (1955)

4. Marczyk, J: Principles of Simulation-Based Computer-Aided Engineering. FIM Publications, Barcelona (1999)

5. Dubois, D, Prade, H: Possibility Theory: An Approach to Computerized Processing of Uncertainty. Plenum, New York (1988)

6. Zadeh, LA: Fuzzy sets. Inform Contr. 8, 338-353 (1965)

7. Zadeh, LA: Fuzzy sets as a basis for a theory of possibility. Fuzzy Set Syst. 1, 3-28 (1978)

8. Degrauwe, D: Uncertainty propagation in structural analysis by fuzzy numbers. PhD Thesis, Katholieke Universiteit Leuven, Leuven, Belgium (2007)

9. Bothe, H-H: Fuzzy Logic: Einführung in Theorie und Anwendungen. Springer, Berlin (1993)

10. Hanss, M, Klimke, A: On the reliability of the influence measure in the transformation method of fuzzy arithmetic. Fuzzy Set Syst. 143(3), 371-390 (2004)

11. Seibel, A, Schlattmann, J: An analytical approach to evaluating monotonic functions of fuzzy numbers. In: Proceedings of the 8th Conference of the European Society for Fuzzy Logic and Technology, Milano, Italy, 11-13 Sept 2013, pp. 289-293 (2013)

12. International Organization for Standardization: ISO 16047: Fasteners_-Torque/Clamp Force Testing. International Organization for Standardization, Geneva (2005)

13. Zou, Q, Zhu, D, Sun, TS, Nassar, S, Barber, GC, El-Khiamy, H: Contact mechanics approach to determine effective radius in bolted joints. J Tribol. 127(1), 30-36 (2005)

14. Hanss, M: Applied Fuzzy Arithmetic: An Introduction with Engineering Applications. Springer, Berlin (2005) 